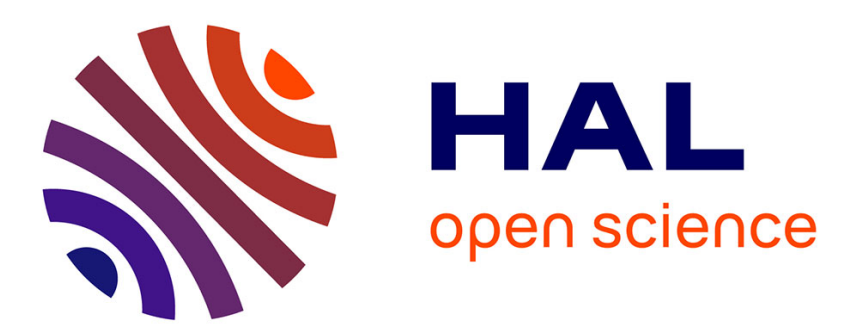

\title{
Passivity Analysis of a motion control for robots manipulators with dynamic friction
}

Carlos Canudas de Wit, R. Kelly

\section{To cite this version:}

Carlos Canudas de Wit, R. Kelly. Passivity Analysis of a motion control for robots manipulators with dynamic friction. Asian Journal of Control, 2007, 9 (9), pp.30-36. 10.1111/j.19346093.2007.tb00301.x . hal-00201787

\section{HAL Id: hal-00201787 \\ https://hal.science/hal-00201787}

Submitted on 2 Jan 2008

HAL is a multi-disciplinary open access archive for the deposit and dissemination of scientific research documents, whether they are published or not. The documents may come from teaching and research institutions in France or abroad, or from public or private research centers.
L'archive ouverte pluridisciplinaire HAL, est destinée au dépôt et à la diffusion de documents scientifiques de niveau recherche, publiés ou non, émanant des établissements d'enseignement et de recherche français ou étrangers, des laboratoires publics ou privés. 


\title{
Passivity Analysis of a Motion Controller for Robot Manipulators with Dynamic Friction
}

\author{
Carlos Canudas de $\mathrm{Wit}^{\dagger}$ and Rafael Kelly ${ }^{\ddagger}$ \\ †Laboratoire d'Automatique de Grenoble \\ URA CNRS 228, ENSIEG-INPG, B.P. 46 \\ 38402, Grenoble, FRANCE \\ e-mail: carlos.canudas-de-wit@inpg.fr \\ †Dirección de Telemática, CICESE \\ Apdo. Postal 2615, Adm. 1 \\ Ensenada, B.C., 22800, MEXICO \\ e-mail: rkelly@cicese.mx
}

\begin{abstract}
This paper addresses the motion control in joint space of robot manipulators with friction described by the generalized LuGre friction model. The paper extends the nonadaptive version of the Slotine and Li's control system originally designed for friction-free manipulators to the case where a friction observer is incorporated to deal with friction. This observer corresponds to an adaptation of the friction compensation scheme proposed in Canudas de Wit et al in 1975. Passivity concepts are the fundamental tools invoked in this paper to analyze the closed-loop system behavior which lead to the conclusion of global asymptotic joint position tracking. A major advantage of this framework is that it allows to develop in a separate way the control law from the observer design provided that each part satisfies some passivity properties.
\end{abstract}

KeyWords: Passivity-based control, Robot control, LuGre friction. 


\section{INTRODUCTION}

Friction is a harmful physical phenomenon present in mechanisms such as in robotic systems and machine tools. Typical undesirable behaviors caused by friction in control systems of mechanisms are steady-state positioning errors and oscillations due to limit cycles. Fortunately, attenuation of some of these effects can be achieved by utilizing friction compensators into the control systems.

Model-oriented friction compensation techniques are based on the knowledge of a suitable friction model that predicts the real friction and commands an opposed control action to compensate it [1]. However, friction is a hard process to model because the many ingredients involved in this physical phenomenon such as material of the bodies in contact, lubrication, temperature, etc. Thus, a large class of simplified friction models are available for control system design. Two main families can be distinguished: static and dynamic models. The former is described by static maps between velocity and friction force (or torque) such as the Coulomb friction and viscous friction models. Although there are many applications using such a models, e.g. [2] and [3], several important properties observed in systems with friction such as stick-slip motion, pre-sliding displacement, Dahl effect and frictional lag, cannot be only explained by static models. This is basically due to the fact that friction does not have an instantaneous response on a change of velocity, i.e., it has an internal dynamics. This is the reason of the dynamic friction models such as the Dahl model [4] and its generalization the LuGre model introduced by [3].

The LuGre model captures the Coulomb and viscous friction terms, Stribeck effect, the stick-slip motion, and pre-sliding displacement. All these static and dynamic characteristics of friction are important for friction compensation purposes. Thus, the LuGre model turns out to be suitable for the design of model-based friction compensation schemes into control systems. The control scheme proposed in [3] uses the inherent passivity property from the velocity to the internal state as a control design tool. In multi-dimensional systems with nonlinear coupling like the robot manipulators, it is some time suited to formulated the control problem in a way that a better passivity property of the friction model are stated from the velocity to the predicted force. For the simplest version of the LuGre model (see [3]) the passivity from velocity to force is limited by an inequality that restrict choice of there parameters; see [5], [6], [7].

If these conditions can be not satisfied for a particular mechanisms, then it is also possible to use the generalized form of the LuGre model introduced in [6] that makes the I/O maps from velocity to force passive for an arbitrarily value set of parameters. The idea is based 
in making the micro-damping term in the original LuGre model, vanish as the velocity increases. In this paper we use such a model to enforce the stability analysis via passivity. These mathematical tools have been also utilized for the study of adaptive control systems of robot manipulators [8], [9].

This paper studies the nonadaptive version of the Slotine and Li's control system introduced in [10] but incorporating an observer-based friction compensator to deal with robots having friction characterized by the generalized LuGre model. This analysis is carried out completely in an input-output analytical framework. An important previous related work was reported by [11] where the Slotine and Li's control structure is considered but two main differences arise with regard to this paper: first, a smooth friction observer is considered here while in [11] a discontinuous action is used instead of a friction observer, and second, we analyze the control system under an input-output optics while in [11] is performed via the Lyapunov theory. Finally, recent works, like the one in [12] addresses similar problems in the sense that the generalized LuGre model is included into the study but differs in the observer gain depending on the state variables (instead of a simple constant gain as proposed in this paper) and more important, without explicit application to the Slotine and Li's control scheme.

In this paper, it will be shown that the overall closed-loop system can be described by the feedback interconnexion of two passive subsystems. One subsystem describes the error tracking dynamics and the other the observer dynamics. This approach allows to analyze separately each of these subsystems and provides global asymptotic position tracking, throughout the only requirement of ensuring the output strictly passivity of the subsystem associated with the error tracking dynamics.

We digress momentarily to recall the mathematical preliminaries borrowed from [13] related to passivity theory useful for the purpose of this paper. We will denote by $R_{+}$the set of nonnegative real numbers, and by $R^{n}$ the $n$-dimensional real vector space over $R$ endowed with the Euclidean norm $\|\cdot\|$. All operators are assumed to be well defined over the extended normed function space $L_{2}^{n}$ defined as

$$
L_{2}^{n}=\left\{x: R_{+} \rightarrow R^{n}: \int_{0}^{T}\|x(s)\|^{2} d s<\infty, \forall T \in R_{+}\right\} .
$$

Consider the operator

$$
\begin{aligned}
\Sigma: L_{2}^{n} & \rightarrow \quad L_{2}^{n} \\
u & \mapsto y=\Sigma(u)
\end{aligned}
$$


The operator $\Sigma$ is said to be passive if there exists a constant $\beta \geqslant 0$ such that

$$
\int_{0}^{T} u(s) y(s) d s \geqslant-\beta \quad \forall T \geqslant 0 .
$$

$\Sigma$ is input strictly passive if there exists a constant $\rho>0$ such that

$$
\int_{0}^{T} u(s) y(s) d s \geqslant \rho \int_{0}^{T}\|u(s)\|^{2} d s \quad \forall T \geqslant 0 .
$$

$\Sigma$ is output strictly passive if there exists a constant $\mu>0$ such that

$$
\int_{0}^{T} u(s) y(s) d s \geqslant \mu \int_{0}^{T}\|y(s)\|^{2} d s \quad \forall T \geqslant 0 .
$$

\section{ROBOT MODEL WITH DYNAMIC LUGRE FRIC- TION}

The dynamic model of a $n$ degrees-of-freedom robot manipulator with friction at the joints is given by [14]:

$$
M(q) \ddot{q}+C(q, \dot{q}) \dot{q}+g(q)+F(\dot{q})=\tau
$$

where $q \in R^{n}$ is the joint position, $M(q)$ is the inertia matrix, $C(q, \dot{q}) \dot{q}$ is the Coriolis and centripetal matrix, $g(q)$ is the gravitational torque vector, $\tau$ in the input torque and $F(\dot{q})$ is the friction torque.

Although a number of friction are available in the literature, the friction torque $F(\dot{q})$ is usually modeled by nonlinear static functions of the velocity $\dot{q}$. However, several friction phenomena cannot be explained with this simple approach. This has motivated the introduction of more adequate dynamic friction model as the LuGre dynamic friction model described by $[3]$

$$
\begin{aligned}
\dot{z} & =\dot{q}-N(\dot{q}) z \\
F & =\sigma_{0} z+\sigma_{1} \dot{z}+\alpha_{2} \dot{q}
\end{aligned}
$$

where

$$
N(\dot{q})=\operatorname{Diag}\left\{\frac{\left|\dot{q}_{i}\right|}{g\left(\dot{q}_{i}\right)}\right\}
$$

and

$$
g\left(\dot{q}_{i}\right)=\frac{1}{\sigma_{0}}\left[\alpha_{0}+\alpha_{1} e^{-\left(\frac{\dot{q}_{i}}{v_{0}}\right)^{2}}\right]
$$


for $i=1, \cdots, n$, and notation $\operatorname{Diag}\{\cdot\}$ stands for a diagonal matrix.

The friction internal state $z \in R^{n}$ describes the averaging deflection (relative) of the contact surfaces during the sticktion phases (this state is not measurable). The complete friction model is thus characterized by the four "static parameters" $\alpha_{0}, \alpha_{1}, \alpha_{2}$ and $v_{0}$, and the two "dynamic" parameters $\sigma_{0}, \sigma_{1}$. In their turn, the former parameters have the following physical meaning: $\alpha_{0}+\alpha_{1}$ represents the static parameter, $\alpha_{0}$ denotes the Coulomb parameter, $v_{0}$ stands for the Stribeck parameter and $\alpha_{2}$ is the viscous parameter.

For reasons of simplicity, but without lost of generality, in this paper we have taken the same friction parameters for each joint. $v_{0}$ is a scalar constant, while the other parameters stand for diagonal matrices of equal coefficients; for instance $\sigma_{0}=\sigma_{0} I$ is a $n \times n$ matrix. The parameter $\sigma_{0}$ can be understood as being a stiffness coefficient of the microscopic deformations of $z[\mathrm{rad}]$ during the pre-sliding displacement, and $\sigma_{1}$ as being the damping coefficient associated with $\dot{z}$. See Fig. 1

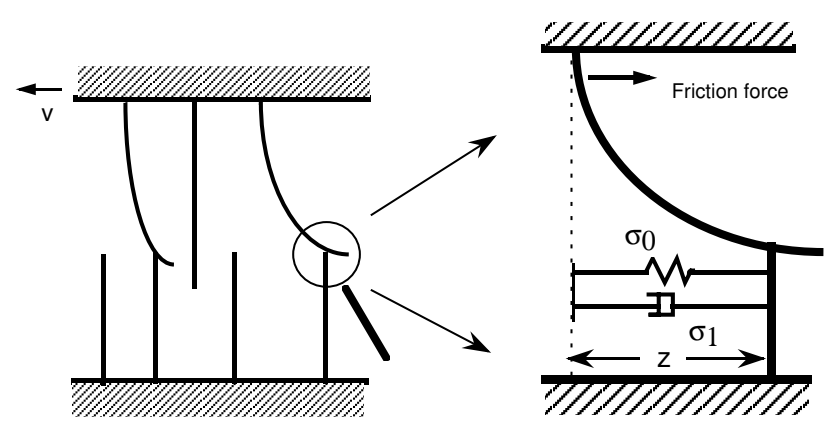

Figure 1: Microscopic representation of surfaces in contact

The scalar (one dimension) LuGre model has been shown in [3] to be passive whether the map $\dot{q} \mapsto z$ is considered. Necessary and sufficient conditions for the passivity of the map $\dot{q} \mapsto F$ have been reported in [7]. This condition is

$$
\sigma_{1} \leqslant \frac{\sigma_{2} \alpha_{0}}{\alpha_{1}}
$$

This inequality imposes an upperbound in the local behavior of the motion within the predisplacement region. The linear approximation in this region for one joint of inertia $J$ is described by the linear map $G(s): \tau \mapsto q$, is:

$$
G(s)=\frac{1}{J s^{2}+\left(\sigma_{1}+\sigma_{2}\right) s+\sigma_{0}} .
$$

Typically one would like to set $\sigma_{1}$ to obtain well-behaved stick-slip transitions, during simulations, i.e.

$$
\sigma_{1}=2 \zeta \sqrt{\sigma_{0} J}-\sigma_{2}
$$


Typically, $\zeta=1$ is chosen, in order to suitable damp the poles of $G(s)$. However, if the passivity property is to be enforced, then the larger damping compatible with the passivity condition is given by

$$
\zeta \leqslant \frac{\sigma_{2}}{2 \sqrt{\sigma_{0} J}}\left[\frac{\alpha_{0}}{\alpha_{1}}+1\right]
$$

This constraint can easily relaxed by using the the extended LuGre model obtained by making each of the $\sigma_{1}$ 's velocity dependent [6], i.e.

$$
\sigma_{1}\left(\dot{q}_{i}\right)=c_{1} e^{-\dot{q}_{i}^{2} / c_{0}^{2}}
$$

where $c_{1}$ and $c_{0}$ are free parameters. The idea is that in pre-sliding when the model need to be well damp, the value of $\sigma_{1} \approx c_{1}$ can be set large enough, whereas $\sigma_{1}$ decreases as velocity increases. It should be notice that large values of $\sigma_{1}$ at large velocities restrict the passivity properties.

The passivity property from $\dot{q} \mapsto F$ as it will be shown below provided that the constants $c_{1}$ and $c_{0}$ are selected such that

$$
\sigma_{0} I-c_{1} \operatorname{Diag}\left\{e^{-\dot{q}_{i}^{2} / c_{0}^{2}}\right\} N(\dot{q})>0, \quad \forall \dot{q}
$$

For a given value of $c_{1}$ (fixing the desired local damp properties), a simple sufficient condition to this hold is to select the positive $c_{0}$ as

$$
c_{0}<e^{1 / 2} \alpha_{0} \sqrt{2 / c_{1}^{2}}
$$

Note that the steady-state characteristic of the model is invariant.

Property 1. Both the LuGre model and the extended LuGre model (2)-(6) have the same static map at constant velocity, which captures the static $\left(\alpha_{0}+\alpha_{1}\right)$, Coulomb $\left(\alpha_{0}\right)$, the Stribeck $\left(v_{0}\right)$ and viscous $\left(\alpha_{2}\right)$ friction coefficients.

Proof. Assume that the velocity $\dot{q}$ is a non zero constant, thus matrix $N(\dot{q})$ defined in (4) is nonsingular. Therefore, according with (2), the steady state value of $z$ is given by

$$
z_{s s}=N(\dot{q})^{-1} \dot{q}
$$

By noting that in steady state $\dot{z}=0$, hence from (3) we get

$$
F_{s s}=\sigma_{0} z_{s s}+\alpha_{2} \dot{q}=\sigma_{0} N(\dot{q})^{-1} \dot{q}+\alpha_{2} \dot{q}
$$


Finally, using (4) and (5) we obtain

$$
F_{s s}=\operatorname{Diag}\left\{\alpha_{0}+\alpha_{1} e^{-\left(\frac{\dot{q}_{i}}{v_{0}}\right)^{2}}\right\} \operatorname{sgn}(\dot{q})+\alpha_{2} \dot{q} .
$$

\section{$\nabla \nabla \nabla$}

The extended LuGre model described by (2)-(6) satisfies the important physical property of passivity. This is summarized in the following

Proposition 1. The extended LuGre dynamic friction model (2)-(6) defines an input strictly passive operator $\dot{q} \mapsto F$, i.e.

$$
\int_{0}^{T} \dot{q}^{T}(t) F(t) d t \geqslant \rho \int_{0}^{T}\|\dot{q}(t)\|^{2} d t
$$

for all $T \geqslant 0$ and some $\rho>0$.

Proof. First, it is convenient to rewrite model (2)-(3) as

$$
\begin{aligned}
\dot{z} & =-N(\dot{q}) z+\dot{q} \\
F & =\left[\sigma_{0} I-\sigma_{1} N(\dot{q})\right] z+\left[\sigma_{1}+\alpha_{2}\right] \dot{q}
\end{aligned}
$$

Notice that matrix $\sigma_{0} I-\sigma_{1} N(\dot{q})$ is positive definite by a proper selection of $c_{1}$, thus the constant $\lambda_{0}$ defined as

$$
\lambda_{0}=\inf _{\dot{q} \in R^{n}} \lambda_{\min }\left\{\sigma_{0} I-\sigma_{1} N(\dot{q})\right\}
$$

is positive.

Let us consider the positive definite function $V(z)=\frac{\lambda_{0}}{2} z^{T} z$. Its time derivative along the trajectories of the model (8)-(9) is given by

$$
\dot{V}(z)=\lambda_{0}\left[z^{T} \dot{q}-z^{T} N(\dot{q}) z\right]
$$

Since matrix $\sigma_{0} I-\sigma_{1} N(\dot{q})$ is positive definite and therefore nonsingular, we substitute $z$ from (9) into (10) to get

$$
\begin{aligned}
\frac{\dot{V}(z)}{\lambda_{0}} & =\dot{q}^{T}\left[\left[\sigma_{0} I-\sigma_{1} N(\dot{q})\right]^{-1}\left[F-\left[\sigma_{1}+\alpha_{2}\right] \dot{q}\right]\right]-z^{T} N(\dot{q}) z \\
& \leqslant \dot{q}^{T}\left[\sigma_{0} I-\sigma_{1} N(\dot{q})\right]^{-1} F-\left[\sigma_{1}+\alpha_{2}\right] \dot{q}^{T}\left[\sigma_{0} I-\sigma_{1} N(\dot{q})\right]^{-1} \dot{q} \\
& \leqslant \frac{1}{\lambda_{0}} \dot{q}^{T} F-\left[\sigma_{1}+\alpha_{2}\right] \lambda_{\min }\left\{\left[\sigma_{0} I-\sigma_{1} N(\dot{q})\right]^{-1}\right\}\|\dot{q}\|^{2}
\end{aligned}
$$


where we have used the fact that $\dot{q}^{T}\left[\sigma_{0} I-\sigma_{1} N(\dot{q})\right]^{-1} F \leqslant \frac{1}{\lambda_{0}} \dot{q}^{T} F$ because $\left[\sigma_{0} I-\sigma_{1} N(\dot{q})\right]^{-1}$ is a diagonal matrix.

Finally, form inequality (11) we have the conclusion that

$$
\begin{aligned}
\int_{0}^{T} \dot{q}^{T}(t) F(t) d t \geqslant & V(z(T))-V(z(0)) \\
& +\frac{\alpha_{2} \lambda_{0}}{\lambda_{1}} \int_{0}^{T}\|\dot{q}(t)\|^{2} d t
\end{aligned}
$$

with $\lambda_{1}=\sup _{\dot{q} \in R^{n}} \lambda_{\operatorname{Max}}\left\{\sigma_{0} I-\sigma_{1} N(\dot{q})\right\}$ and $\rho=\frac{\alpha_{2} \lambda_{0}}{\lambda_{1}}$.

$\nabla \nabla \nabla$

Remark: Note that this property establishes the stronger input strictly passivity property of the map $\dot{q} \mapsto F$, than the weaker passivity evoked in [6].

\section{CONTROL SYSTEM ANALYSIS}

Let us denote by $q_{d}$ the desired position which is assumed to be twice differentiable and $\tilde{q}=q-q_{d}$ stands for the tracking position error. Assuming that all the robot parameters are known, the nonadaptive version of the Slotine and Li's control law can be written as [10]

$$
\begin{aligned}
\tau= & M(q)\left[\ddot{q}_{d}-\Lambda \dot{\tilde{q}}\right]+C(q, \dot{q})\left[\dot{q}_{d}-\Lambda \tilde{q}\right]+g(q) \\
& -K_{v} s+\hat{F}
\end{aligned}
$$

where $s=\dot{\tilde{q}}+\Lambda \tilde{q}$, and $K_{v}, \Lambda$ are diagonal positive definite matrices, and $\hat{F}$ is the output of the friction observer given by

$$
\begin{aligned}
\dot{\hat{z}} & =-N(\dot{q}) \hat{z}+\dot{q}-K s, \\
\hat{F} & =\sigma_{0} \hat{z}+\sigma_{1} \dot{\hat{z}}+\alpha_{2} \dot{q},
\end{aligned}
$$

with $K$ a diagonal positive definite matrix.

The introduction of the friction observer (13)-(14) is motivated by the fact that the neither friction force $F$, nor the variable $z$ are measured, and hence they need to be predicted.

Let us define the error variables $\tilde{z}=z-\hat{z}$ and $\tilde{F}=F-\hat{F}$. The closed-loop equation is obtained by substituting the control law (12)-(14) into the robot dynamics (1)-(6), giving

$$
M(q) \dot{s}=-C(q, \dot{q}) s-K_{v} s-\tilde{F}
$$




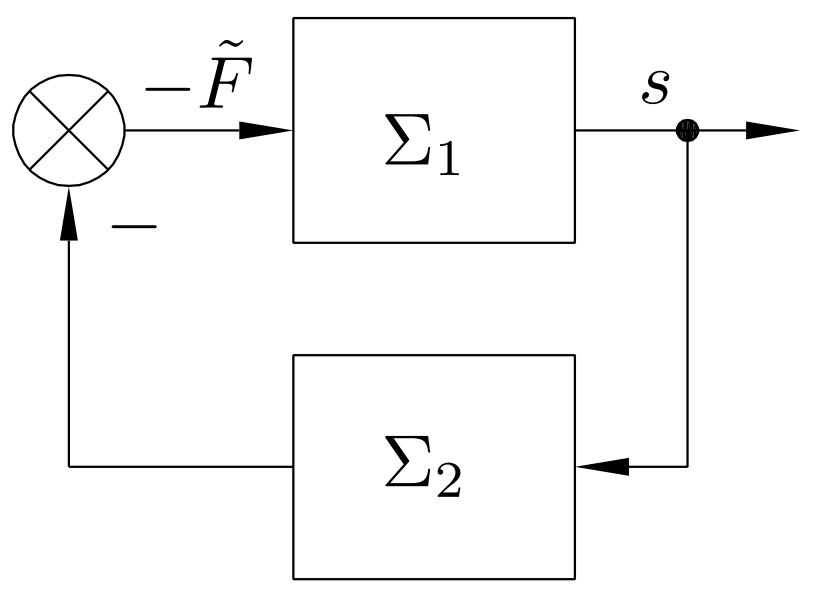

Figure 2: Feedback interconnection of two strictly K passive subsystems.

$$
\begin{aligned}
\dot{\tilde{z}} & =-N(\dot{q}) \tilde{z}+K s \\
\tilde{F} & =\sigma_{0} \tilde{z}+\sigma_{1} \dot{\tilde{z}} \\
& =\left[\sigma_{0} I-\sigma_{1} N(\dot{q})\right] \tilde{z}+\sigma_{1} K s .
\end{aligned}
$$

The closed-loop system can be seen as the feedback interconnexion of two subsystems as indicated in Fig. 2.

The first subsystem, $\Sigma_{1}$, is given by (15) which defines the operator $\Sigma_{1}:-\tilde{F} \mapsto s$. The input-output property is summarized in the following

Proposition 2. The operator $\Sigma_{1}:-\tilde{F} \mapsto s$ defined by (15) is output strictly passive, that is,

$$
\int_{0}^{T}-\tilde{F}(t)^{T} s(t) d t \geqslant \mu \int_{0}^{T}\|s(t)\|^{2} d t
$$

for all $T \geqslant 0$ and $\mu>0$.

Proof. In the context of the input-output analysis of the Slotine and Li's adaptive controller, [15] studied the system

$$
M(q) \ddot{q}_{r}=-C(q, \dot{q}) \dot{q}_{r}-K_{v} \dot{q}_{r}-Y \tilde{a}
$$

where $Y \tilde{a}$ can be seen as the input and $\dot{q}_{r}$ as the output. They proved for the first time that the map $Y \tilde{a} \mapsto \dot{q}_{r}$ is output strictly passive. Since the structure of $\Sigma_{1}$ is exactly the same, then the passivity result follows straightforwardly. 
The second subsystem, $\Sigma_{2}$ is described by (16)-(17) and depends only on the observer structure (13)-(14). This defines the operator $\Sigma_{2}: s \mapsto \tilde{F}$. Its input-output property is established in the following

Proposition 3. The operator $\Sigma_{2}: s \mapsto \tilde{F}$ defined by (16)-(17) is passive, that is,

$$
\int_{0}^{T} s^{T}(t) \tilde{F}(t) d t \geqslant-\beta
$$

for all $T \geqslant 0$ and some $\beta \geqslant 0$.

Proof. The proof shown below follows similar steps than those used in the proof of Proposition 1.

Consider the following positive definite function

$$
V_{2}(\tilde{z})=\frac{\lambda_{0} \lambda_{2}}{2} \tilde{z}^{T} \tilde{z}
$$

where $\lambda_{2}=\lambda_{\min }\left\{K^{-1}\right\}$. Its time derivative along the trajectories of (16)-(17) yields

$$
\dot{V}_{2}(\tilde{z})=\lambda_{0} \lambda_{2}\left[\tilde{z}^{T} K s-\tilde{z}^{T} N(\dot{q}) \tilde{z}\right] .
$$

Since matrix $\sigma_{0} I-\sigma_{1} N(\dot{q})$ is positive definite, we substitute $\tilde{z}$ from (17) into (20) to get

$$
\begin{aligned}
\frac{\dot{V}_{2}(\tilde{z})}{\lambda_{0} \lambda_{2}}= & s^{T} K\left[\sigma_{0} I-\sigma_{1} N(\dot{q})\right]^{-1}\left[\tilde{F}-\sigma_{1} K s\right]- \\
& -\tilde{z}^{T} N(\dot{q}) \tilde{z} \\
\leqslant & s^{T} K\left[\sigma_{0} I-\sigma_{1} N(\dot{q})\right]^{-1} \tilde{F}- \\
& \sigma_{1} s^{T} K\left[\sigma_{0} I-\sigma_{1} N(\dot{q})\right]^{-1} K s \\
\leqslant & \frac{1}{\lambda_{0} \lambda_{2}} s^{T} \tilde{F}
\end{aligned}
$$

and finally we conclude the passivity of the friction observer subsystem $\Sigma_{2}$, i.e.,

$$
\begin{aligned}
\int_{0}^{T} s^{T}(t) \tilde{F}(t) d t & \geqslant V_{2}(\tilde{z}(T))-V_{2}(\tilde{z}(0)) \\
& \geqslant-V_{2}(\tilde{z}(0))
\end{aligned}
$$

where it can be identified from (19) that $\beta=V_{2}(\tilde{z}(0))$. 
The closed-loop system (15)-(17) is thus described by the feedback interconnexion of an output strictly passive operator $\Sigma_{1}$ with an passive operator $\Sigma_{2}$. The main result regarding this feedback system is stated in the following

Proposition 4. The feedback interconnexion of the output strictly passive operator $\Sigma_{1}$ : $-\tilde{F} \mapsto s$ and the passive operator $\Sigma_{2}: s \mapsto \tilde{F}$ leads to

- $s \in L_{2}^{n}$

- $\lim _{t \rightarrow \infty} \tilde{q}(t)=0$

Proof. Passivity theorems are available to analyze the input-output stability properties of this kind of interconnected passive systems [16]. However, for the sake of completeness we provide below a simple self-contained alternative proof.

From the friction observer subsystem property (19), we have

$$
\beta \geqslant \int_{0}^{T}-s^{T}(t) \tilde{F}(t) d t .
$$

Incorporating this into (18) yields

$$
\begin{aligned}
\beta & \geqslant \int_{0}^{T}-s^{T}(t) \tilde{F}(t) d t \\
& \geqslant \mu \int_{0}^{T}\|s(t)\|^{2} d t
\end{aligned}
$$

hence

$$
\frac{\beta}{\mu} \geqslant \int_{0}^{T}\|s(t)\|^{2} d t
$$

for all $T \geqslant 0$.

Since $\mu$ and $\beta$ are positive constants, then we conclude that $s \in L_{2}^{n}$. Now from the definition of $s$ we have the following relation

$$
\tilde{q}=[p I+\Lambda]^{-1} s
$$

where $p=d / d t$ stands for the differential operator and $I$ for the identity matrix. This is a strictly proper exponentially stable linear system whose input $s$ belongs to $L_{2}^{n}$. The output $\tilde{q}$ of such a system must vanish asymptotically [13], that is

$$
\lim _{t \rightarrow \infty} \tilde{q}(t)=0 .
$$


This proves the global asymptotic position tracking.

\section{CONCLUSIONS}

One of the most popular motion control techniques for robot manipulators in joint space is the so-called Slotine and Li's control scheme (nonadaptive version). In order to better perform in practice, friction compensators may be incorporated to such control system. The extended LuGre friction model, which matches the physical requirement of being a passive phenomenon, allows the design of a friction observer retaining also key passivity properties.

Exploiting the fact that the Slotine and Li's controller enjoys of nice passivity properties, this paper has carried out the closed-loop system behavior in a complete input-output framework. It is shown that the overall closed-loop system can be described by the feedback interconnexion of two passive subsystems. One of them related to the robot/controller structure - thus a passivity based control scheme - and the remaining to the friction observer. The analysis is based on standard passivity tools to get the desired conclusion of global asymptotic position tracking.

The input-output approach presented in this paper offers a framework to analyze also other control law structures together with the friction observer described here. The only requirement for global asymptotic position tracking is to ensure the output strictly passivity of the subsystem associated with such control laws.

\section{References}

[1] Armstrong-Helouvry, B., P. Dupont and C. Canudas de Wit, "A Survey of Analysis Tools and Compensation Methods for the control of Machines with Friction", Automatica, Vol. 30, No. 7, pp.1083-1138, (1994).

[2] Canudas de Wit, C., K.J. Åström and K. Braun, "Adaptive Friction Compensation in DC Motor Drives", IEEE Journal of Robotics and Automation, Vol. RA-3, No. 6, pp. 681-685, (1987).

[3] Canudas de Wit, C., H. Olsson, K.J. Åström, and P. Lischinsky, "A New Model for Control of Systems with Friction", IEEE Trans. on Automatic Control, Vol. 40, No. 3, pp.419-425, (1995). 
[4] Dahl, P.R., "Solid friction damping of mechanical vibrations", AIAA Journal, Vol. 14, No. 12, pp. 1675-1682, (1976).

[5] Gäfvert, M., Comparison of two friction models, Master's thesis LUTFD2/TFRT-5561SE, Lund Institute of Technology, Sweden, page 53, property 3.7, (1996).

[6] Olsson, H., Control systems with Friction, Ph. D. Thesis, Lund Institute of Technology, Sweden, (1996).

[7] Baravanov, N. and R. Ortega, "Necessary and sufficient conditions for passivity of the LuGre model", IEEE Trans. on Automatic Control, Vol. 45, No. 4, pp. 830-832, (2000).

[8] Kelly, R., R. Carelli and R. Ortega, "Adaptive motion control design of robot manipulators: An input - output approach", International Journal of Control, Vol. 50, No. 6, pp. 2563-2581, (1989).

[9] Ortega R. and M. W. Spong, "Adaptive motion control of rigid robots: A tutorial", Automatica, Vol. 25, No. 6, pp. 877-888, (1989).

[10] Slotine, J. J. and W. Li, "Adaptive manipulator control: A case study", IEEE Transactions on Automatic Control, vol. AC-33, No. 11, pp. 995-1003, (1988).

[11] Panteley, E., R. Ortega and M. Gäfvert, "An adaptive friction compensator for global tracking in robot manipulators", Systems and Control Letters, Vol. 33, pp. 307-313, (1998).

[12] Shiriaev, A., A. Robertsson and R. Johansson, "Friction compensation for passive systems based on the LuGre model", 2nd IFAC Workshop on Lagrangian and Hamiltonian Methods for Nonlinear Control, Seville, pp. 183-188, (2003).

[13] Desoer, C., and M. Vidyasagar, Feedback systems: Input-Output properties, Academic Press, (1975).

[14] Spong, M. W. and M. Vidyasagar, Robot dynamics and control, John Wiley and Sons, (1989).

[15] Kelly, R. and R. Ortega, "Adaptive control of robot manipulators: An input-output approach", Proc. of the IEEE Int. Conference on Robotics and Automation, Philadelphia, PA., pp. 699-703, (1988).

[16] Khalil, H. K., Nonlinear systems analysis, Prentice Hall, Second Edition, (1996). 